\title{
GESTIÓN DE DESEMPEÑO SOCIAL EN LAS MICROFINANZAS
}

\section{SOCIAL PERFORMANCE MANAGEMENT IN MICROFINANCE}

\section{Referencia del artículo}

Aldana, M. (2020). Gestión de desempeño social en las microfinanzas. Revista Científica del SEP. 3(I), 5I-57.

DOI: https://doi.org/10.36958/sep.v3i01.35

\author{
Marvin Amilkar Aldana Bolaños \\ marvinamilkar@gmail.com \\ Licenciado en Económicas, Estudiante de Maestría en GerenciaAdministrativa de Recursos Humanos \\ Centro Universitario de Sur-Oriente CUNSURORI, Universidad deSan Carlos de Guatemala \\ Fecha de recibido:10/11/2019 \\ Fecha de aceptado: 06/06/2020
}

\section{RESUMEN}

La gestión del desempeño social es la ecuación perfecta para las organizaciones de hoy en dí-a; derivado a que, con la práctica idónea se obtendrá un balance perfecto del desempeño financiero y social por consiguiente un enfoque equilibrado que beneficia al colaborador, a los clientes y a la organización de forma integral, debido a que sus procesos y resultados no pueden ser alterados porque están sumamente vinculados con la filosofí-a y valores institucionales.

\section{PALABRAS CLAVE}

Gestión del desempeño social, responsabilidad social, innovación, inteligencia y principios de protección al cliente

\section{ABSTRACT}

Social performance management is the perfect equation for today's organizations; derived from the fact that, with the ideal practice, a perfect balance of financial and social performance will be obtained, consequently a balanced approach that benefits the collaborator, the clients and the organization in an integral way, because its processes and results cannot be altered because they are highly linked to institutional philosophy and values.

\section{KEYWORDS}

Management of social performance, social responsibility, intelligence and customer protection principes 


\section{INTRODUCCIÓN}

Durante muchos años las organizaciones han diseñado una misión, visión y valores institucionales dentro de su plan estratégico y/u procesos, con el objetivo de perfilar un trayecto de que se hace, a donde va y como ejecuta, sin causar daño y obtener utilidades; sin embargo resulta que la globalización hoy en día atrapa a las estructuras de tal manera que la filosofía y las políticas de calidad se empiezan a modificar sin darse cuenta que por alcanzar las metas institucionales se deja de poner en práctica todo aquello que dio origen.

La gestión del desempeño debe proveer una responsabilidad social efectiva y eficiente para mantener el equilibrio idóneo entre el segmento social y financiero y que los procesos estén diseñados para generar valor agregado y así contribuir con un desarrollo sostenido; pero para lograr una adecuada inclusión financiera las organizaciones deben de enfocar sus esfuerzos en las partes interesadas y proveer como un aliado de formula el servicio de protección al cliente, que van desde el diseño de productos y canales de distribución apropiada, prevención del sobreendeudamiento, transparencia, precios responsables, trato justo y respetuoso, privacidad de los datos y mecanismos para la resolución de reclamos.

La información obtenida del desempeño social no solo es útil para la toma de decisiones operacionales y estratégicas sino que es absolutamente necesaria para acompañar al cliente en una ruta de desarrollo y que las organizaciones estén orientadas y convencidas que se debe de promover el servicio financiero no solo al segmento con mayores posibilidades económicas sino a todo aquel cliente que este fuera del segmento crediticio y con condiciones vulnerables para poder aportar desarrollo y velar por su crecimiento continuo.

El objetivo del artículo es analizar la evolución y el desarrollo de la implementación de estándares universales en relación a la gestión del desempeño social, en una empresa financiera líder en Guatemala, con más de 100 agencias, con una cobertura geográfica que asegura estar más cerca de sus clientes, con una colaboración de empleados mayor a 1,000 y una cartera productiva de más de un millardo; así mismo de forma integral que es la gestión de desempeño social en las micro-financieras y cuáles son sus aliados estratégicos para llevar a cabo esta inclusión en los segmentos empresariales con base a escritores y autores.

\section{GESTIÓN DEL DESEMPEÑO SOCIAL}

El reto empieza con definir los enfoques y mejores prácticas de desarrollo que no consideren sólo los aspectos cuantitativos y se empiecen a incluir los cualitativos; los cuales deben tener un vínculo integral con la filosofía de la organización y así transmitir, enfocar, trasladar y sustentar desarrollo en las áreas más vulnerables de la sociedad. La necesidad de políticas centradas en el esfuerzo compartido, abordar la cuestión del desarrollo local exige una lectura crítica de los segmentos estipulados para una buena gestión del desempeño social y que no limita o excluya segmentos regionales, nacionales o global, por el contrario sean incluidos a la sociedad para el servicio y el desarrollo. (Kliksberg, 1997).

Para Di Pietro (1996), emprender la gestión del desarrollo no es simple y sencillamente un deseo de querer apoyar; derivado a que se necesita un mapa general de todas las guías, principios, regulaciones, reglamentos $y$ avances de las grandes corrientes del pensamiento que poco a poco han estructurado un control robusto que diagnostique que; para indicar y/o manifestar que la institución aplica gestión de desempeño social en sus procesos es porque ha realizado un cambio radical en su filosofía y planeación estratégica basada en el cliente y que dicha gestión genere resultados que aproximen al tema y que condicionen la buenas prácticas en forma integral.

En cuanto a los aportes de los autores citados, se encuentra coincidencia en lo siguiente: la inclusión de la gestión de desempeño social en los procesos no es un medio que tiene como objetivo final solo apoyar a un segmento de la sociedad; derivado a que si se enfoca en las microfinancieras, estás deben de otorgar un préstamo y analizar si con el mismo se logrará desarrollo y posicionar al cliente en una zona menos vulnerable y/o de menos pobreza; porque una buena gestión, no excluye a aquella sociedad que no tiene recursos, más bien busca aleados en cuanto a protección al cliente y responsabilidad social y así crear programas que ayuden a lograr la relación financiero y social que tanto se busca en la actualidad.

Por lo consiguiente la cartera atendida esta fraccionada en programas de bancomunales, microempresarios, pequeñas empresas, viviendas y educación; con un registro del $93 \%$ de los clientes con medición de probabilidad de pobreza. Del total de clientes activos el $87 \%$ se ubica en el área rural y el $13 \%$ en el área urbana (FGE, 2017).

\section{POLÍTICAS SOCIALES}

Estas son acciones que utiliza el Estado como forma de intervención en la sociedad civil. En un sentido más exclusivo, es el esquema de acción del Estado en materia social, dirigido a realizar algún cambio en las estructuras sociales de una sociedad sin inclusión financiera. Los orígenes de las políticas sociales nacen con el objetivo de inculcar moral en la economía, a fin de evitar las injusticias derivado las consecuencias sociales de la globalización y/o revolución industrial; por lo consiguiente está se fundamentó, diseño y aprobó por todas aquellas 
personas amenazadas por la pobreza, ancianos, vagabundos y enfermos tengan una mejor oportunidad y protección ante las economías irresponsables (Giron, 2006).

Así mismo Wardle (20l4), plantea que una economía responsable, significa que las finanzas van desde la entrega de servicios financieros de forma transparente, justa, segura y con un enfoque cimentado en la búsqueda incansable de la generación de beneficios y que no excluye segmento, porque dentro de dicha inclusión financiera deben de participar los clientes pobres. Del mismo modo los clientes son protegidos por las buenas prácticas establecidas en los estándares universales en el cuadrante cuatro "Tratar a los clientes responsablemente", que concentra como tratar a los clientes idóneamente y por lo tanto una adecuada gestión para el balance financiero-social, mismo es remunerado como incentivo para las organizaciones con la obtención de la certificación the Smart Compaing.

Derivado a que las condiciones de vida no han mejorado significativamente, la intervención del Estado también está apoyada por entes internacionales; tales como: ONU, The Smart Compaing, Truelift, entre otras que se dedican a impulsar las buenas prácticas de las políticas sociales y como llevarlas a cabo inmersas en procesos integrales que no desvirtúan los resultados, porque toda aquella organización que cuente con políticas sociales está obligada a cambiar su enfoque filosófico si en todo caso sus inicios no eran los esperados y como motivación los certifican como empresas y/u organizaciones con aportes a la sociedad.

Tal es caso de la empresa líder de micro finanzas en el país en: cartera, gestión social y geo posición con el objetivo de llegar a los clientes que poseen una inclusión financiera; que con un arduo trabajó de planificación integral, desarrolló una serie de proyectos de innovación y mejora integral que contribuyen a una ruta de desarrollo sostenido que implica que todo cliente debe salir de extrema pobreza a pobreza y de pobreza a muy vulnerables a la pobreza y así sucesivamente hasta alcanzar la probabilidad de no ser pobres; para que la medición sea objetiva debe poseer cruces de todo tipo; Ejemplo: por clientes, programas, número de operaciones, ubicación geográfica, capacitados, género, edad, etnia, negocio y servicios básicos.

\section{RESPONSABILIDAD SOCIAL}

Este concepto es aplicado específicamente por las grandes empresas, de cualquier sector económico o índole que obliga a cumplir con la filosofía creada, aunque también en empresas, públicas y privadas, incluidas las pequeñas y medianas empresas - pymes y las cooperativas. La realidad es que el criterio de voluntariedad no obliga legalmente a las organizaciones a ser socialmente responsables; pero el mercado sí, derivado a que la sociedad ha aprendido a diferenciar entre empresas comprometidas con causas sociales y aquellas que no lo están y que lejos de aportar destruyen (Correa, 2007).

La financiera líder en micro-finanzas con cobertura nacional, tiene claro el daño que genera a la sociedad un financiamiento mal otorgado es por eso que la protección a sus clientes es primordial y en consecuencia se practican principios de protección al cliente por medio de un diseño y distribución apropiada de productos, prevención del sobreendeudamiento, transparencia, precios responsables, trato justo y respetuoso, privacidad de los datos y mecanismos para la resolución de quejas y reclamos.

\section{INNOVACIÓN DE LA RESPONSABILIDAD SOCIAL}

En general la innovación obtiene un énfasis en la mediación entre la responsabilidad social, las empresas y el éxito competitivo. La innovación aumentará cuando la empresa sea responsable en todos los aspectos, tanto financieros como sociales la innovación convertirá sus gestiones y/u operaciones en una ruta balanceada hacía el éxito rápidamente con el objetivo de potenciar el efecto que por sí misma ya ejercía en la competitividad de la entidad.

Se generan nuevas necesidades que conllevan a, comprender los factores que influyen en las necesidades de cada uno de los usuarios y así poder crear escenarios o parámetros para nunca desequilibrar el enfoque de actividades en relación a su filosofía (Gallardo y Sánchez, 2013).

Para León, et al (20I2) la innovación se torna en las empresas día con día y empieza a ejercer importancia y no sólo en relación a la generación de productos, servicios y procesos que logren mayores resultados, sino más bien cuando los resultados van con un enfoque social es esencial la innovación empre $\neg$ sarial; porque está debe transcender y con ello contribuir con el desarrollo de las sociedades y así buscar oportunamente las brechas y/o falencias de los procesos con el fin de reducir el desfase filosófico, es por eso que la innovación incide en las diversas funciones $y$ procesos organizacionales que marcan ventajas contra otras organizaciones que no cuentan con indicadores que guíen su trayecto de ruta de desarrollo.

La innovación de la financiera líder empieza con la expansión social, fidelización y motivación de los colaboradores con un desempeño social; pero para que esto se haga realidad nace un canal de capacitación constante y adquisición de tecnología de punta enfocada en los mercados, que deja atrás el papel y apoyado de teléfonos inteligentes poder levantar datos en línea para la estandarización de procesos; mismos que permiten ser oportunos, eficientes y responsables en las necesidades y/o demandas exigidas por la expansión de mercado. 


\section{EVOLUCIÓN Y TENDENCIA DE LA RESPONSABILIDAD SOCIAL}

Al respecto Gómez y Quintanilla (20I2), agregan que:

Los informes sistemáticos que buscan entregar datos cuantitativos y cualitativos que den cuenta del comportamiento social, medioambiental y económico de las empresas. Este nombre tiene gran aceptación en España, donde el concepto de memoria ha estado asociado a las revelaciones o notas a la información financiera. En un contexto internacional más amplio, estos informes han recibido también la denominación de Informes de Responsabilidad Social o Informes de Desarrollo Sostenible.

La responsabilidad social no llega a todas las financieras; pero actualmente se ha integrado en el mercado económico de tal forma que si las organizaciones desean limitar su riesgo de crédito deben de considerar las buenas prácticas deseadas en una gestión de desempeño. Claro está que la dificultad de mayor grado de hoy en día es la competencia; misma que en ocasiones se torna desleal o descontrolada por los prestamistas no regulados con tasas de servicios no regulados. No obstante la empresa líder de micro finanzas en Guatemala ha resuelto y/o evolucionado; derivado a la protección de clientes que posee por medio de la dimensión cuatro de los estándares universales para la gestión del desempeño social.

\section{INTELIGENCIA SOCIAL EN LAS ORGANIZACIONES}

A priori al desarrollo es importante revisar algunos elementos a tener en cuenta, con el fin de contextualizar la temática central; derivado a que las variables socioeconómicas son fundamentales para que la inteligencia social de las organizaciones y así empiecen a ejercer y/o aplicar los indicadores y medidas correctivas que sean pertinentes, y por revisar los objetivos fijados en el año 2000 por 189 países se han definido para el milenio; A continuación se detallan las siguientes (ONU, 2000).

I. Erradicar la pobreza extrema y el hambre.

2 Lograr enseñar primaria universal.

3. Promover la igual entre los sexos y el empoderamiento de la mujer.

4. Reducir la mortalidad de los niños menores de 5 años.

5. Mejorar la salud materna.

6 Combatir el VIH/Sida, la malaria y otras enfermedades.

7. Garantizar la sostenibilidad del medio ambiente

8. Fomentar una alianza mundial para el desarrollo.

De lo anterior, se hace referencia que si se tomara como base principal el objetivo primero y las organizaciones se dedicaran a erradicar la pobreza extrema y el hambre encontrarían las siguientes aseveraciones:

I. El mercado laboral ya no proporciona las condiciones mínimas necesarias.

2 Pérdida de trabajo y/o disminución de la fuente hace que cada día más personas se han visto forzadas a aceptar empleos vulnerables y/o denigrantes.

3. Más trabajadores y sus familias están viviendo en pobreza extrema porque los ingresos son menores a los gastos.

4. En la mayoría de las regiones, el progreso para erradicar el hambre se ha estancado.

5. Personas han tenido que abandonar sus hogares por delincuencia y/o persecuciones.

\section{SITUACIÓN ACTUAL}
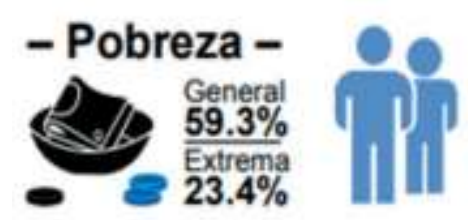

Tasa de Informalidad de empleo $69.8 \%$ 200 mil jovenes $x$ afo ingresan a ta fuerza laboral
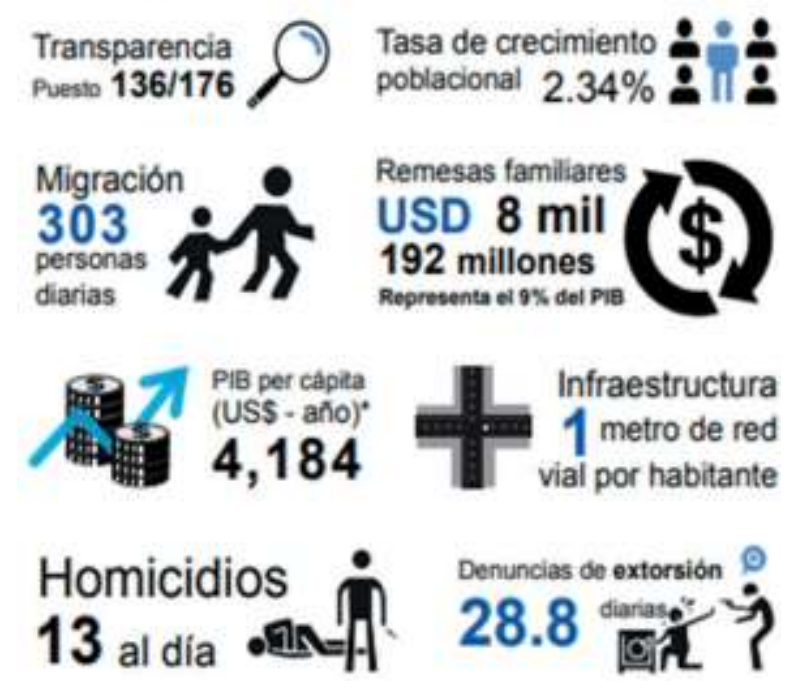

(FMI World Economic, Database, Outlook, Banguat, 2017, citado por Fundación Génesis empresarial en memorial de labores, 2018).

Si bien es cierto, que los objetivos del milenio fueron diseñados en su momento, como una guía de ruta de desarrollo para alcanzar una balanza entre lo social y financiero y que estos fueran de forma sostenible, la realidad es otra derivado a que la situación muestra un escenario delicado en relación a la calidad de vida de las personas (Schvarstein, 20I2).

Es por eso que las organizaciones han identificado que todo lo que se mide se puede mejorar $y$ al mejorar se logran los objetivos institucionales; la financiera líder en micro-finanzas en Guatemala, tiene claro que en sus procesos deben de existir estadísticas, indicadores y procesos tecnológicos definidos con enfoques hacía la mejora continua; derivado a que hoy 
el éxito radica cuando se tiene una guía de comportamientos, necesidades y planes acción para atender de mejor manera su filosofía.

\section{PRINCIPIOS DE PROTECCIÓN AL CLIENTE}

Las brechas más frecuentes entre los principios es la falta de formalización y cultura; porque cada uno de los colaborares no hace por cumplir las buenas prácticas. Las instituciones financieras, no siempre poseen políticas escritas para tratar temas que garanticen las buenas prácticas de protección al cliente; por ejemplo cobranzas, renovaciones de contratos. Del mismo modo, en algunos casos donde si existen las políticas, no hay prácticas que las refuercen, como capacitación de personal, seguimiento, sanciones y acuerdos de servicios. Es por eso que las organizaciones que complementan las prácticas existentes con políticas escritas tienden a desempeñarse significativamente mejor que la mayoría que no los poseen. (Guzman, 20I3).

Por lo anterior la financiera líder ha diseñado un plan, mismo que no es negociable en su incumplimiento; derivado que sus colaboradores practican la filosofía, valores y principios de protección al cliente segmentado en la dimensión No. 4 tratar a los clientes responsablemente de los estándares universales para la gestión del desempeño en un 100\%; mismos que abarcan el diseño y distribución de canales apropiados, prevención del sobre-endeudamiento, transparencia, precios responsables, trato justo y respetuoso, privacidad de los datos y mecanismos para la resolución de quejas y reclamos. Porque dichos procesos están sumamente controlados por auditorias recurrentes, que garantizan y salvaguardan que los clientes reciben los servicios como han sido diseñados y todas aquellas falencias identificadas son corregidas por medio de capacitaciones, sanciones y/o desvinculaciones según sea el caso.

\section{ESTÁNDARES UNIVERSALES PARA LA GESTIÓN DEL DESEMPEÑO SOCIAL DEL SPTF}

Son estándares universales reconocidos y aceptados a nivel internacional que con su práctica en conjunto constituyen una gestión de desempeño social, misma que recae como un recurso fundamental e indispensable para todas aquellas micro financiera que buscan alcanzar metas sociales y que su filosofía prevalezca en el tiempo sin desvirtuarse por factores diversos que inyecta la globalización y con ello pueda lograse resultados sociales que ayudan a la inclusión financiera todos los segmentos más vulnerables del planeta.

Así mismo contribuye con la credibilidad o reputación; los estándares universales están organizados en seis dimensiones y cada dimensión contiene múltiples estándares para asegurar que la práctica es esencial (STPF, 2019).

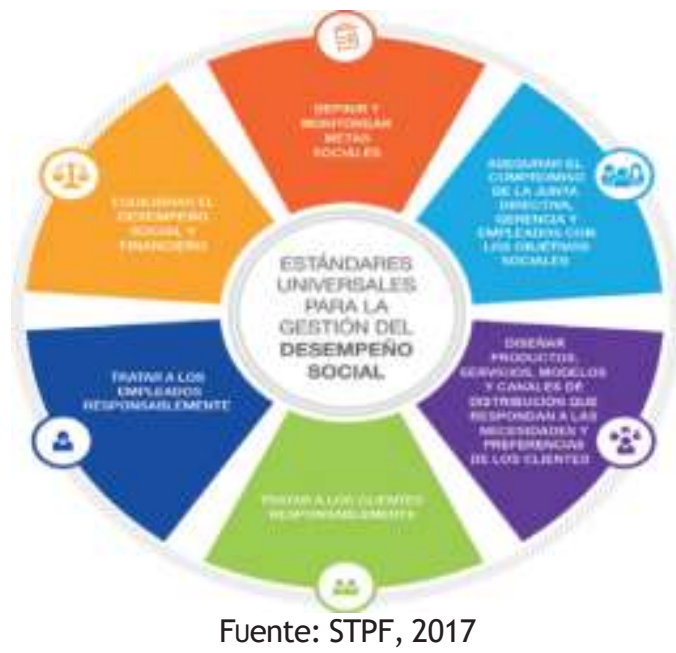

\section{CONCLUSIONES}

Derivado de los antecedentes y análisis teóricos realizados permite acumular abstractos de cómo se debe estructurar el tema de gestión del desempeño social en las micro financieras $y$ es que efectivamente las organizaciones y/o entidades que emplean la gestión del desempeño social generan valor agregado en sus procesos; derivado a que sus clientes internos (empleados), se identifican de mejor manera con la misma y por lo consiguiente aportan eficiencia, eficacia y contribuyen a que las buenas prácticas se cumplan metódicamente de tal forma que las políticas gubernamentales velen para resolver las necesidades de la población, estándares internacionales sean ejecutadas sin desvirtuar el origen, reglamentos internos que hagan cumplir la filosofía de la institución y así solo tengan como objetivo apoyar, desarrollar e incluir al segmento económico.

Dicha inclusión está dirigida a todo aquel cliente externo que este en el segmento vulnerable y/o de extrema pobreza. $Y$ por otro lado los clientes que no estén en extrema pobreza trazarles una ruta de desarrollo sostenida que los lleve a una probabilidad de no ser pobres.

La gestión del desempeño social está íntegramente ligada con la innovación, responsabilidad social y principios de protección al cliente, por lo que toda organización que desee poner en práctica la ecuación del éxito en relación a la balanza financiera y social debe empezar con la medición de su estructura y/o filosofía y si la misma está diseñada para soportar el servicio no dañino que seguramente los estándares internacionales le exigirán.

En general la tendencia de la gestión del desempeño social es hacia la alza por ser un instrumento que basa su certificación en proteger a las partes interesada bajo un enfoque social; derivado a que las instituciones financieras han identificado que entre más cautelosos sean para brindar el servicio, este será de mejor calidad y la población será atendida de igual manera por varios postulantes y así evitar niveles de endeudamiento no deseados que pongan en riesgo la continuidad del negocio. 
La implementación de la gestión del desempeño social es la primera etapa para proteger al cliente derivado que dicho procedimiento certifica que las organizaciones que ejecutan dicha gestión dentro de su planificación y objetivos institucionales el centro de ejecución está basado en el cliente y por lo tanto en cumplir los principios de protección al cliente que busca como resultado una responsabilidad social sostenida llena de oportunidades para la sociedad en las diferentes etapas y/o estatus económicos.

\section{REFERENCIAS BILIOGRÁFICAS}

Correa, J. (2007) "Evolución histórica de los conceptos de responsabilidad social empresarial y balance social" Semestre económico volumen 10 pp 87-102, Medellín, Colombia.

Gallardo, D. Y M. Sánchez (2013) “Análisis de la incidencia de la responsabilidad social empresarial en el éxito competitivo de las microempresas y el papel de la innovación” UniversariaBussines Review, España.

Di Pietro, L. (1996) “Desarrollo e identidades culturales: un desafío en las políticas públicas" en cuadernos americanos, México, UNAM, No. 60.

Giron, Y. (2006) La gestión social y el trabajo social, trabajo de graduación de licenciatura trabajo social, Universidad de San Carlos de Guatemala, Guatemala.

Gómez, M. Y D. Quintanilla (20I2) “Los informes de responsabilidad social empresarial: su evolución y tendencias en el contexto internacional y colombiano" informe final de proyecto de investigación, Facultad de ciencias económicas, Universidad Nacional de Colombia.

FGE (Fundación Génesis Empresarial) (2017), Segmentación de clientes activos, memoria de labores [página web]. Consultado: 19 de septiembre de 2019, http://www.genesisempresarial.org/ nosotrosfundación/.

FGE (Fundación Génesis Empresarial) (2018), Segmentación de clientes activos, memoria de labores [página web]. Consultado: I de septiembre de 2019, http://www.genesisempresarial.org/ nosotrosfundacion/.

Guzmán, S. (20I3) "Estudio sobre las prácticas de protección al cliente en América Latina y el Caribe", The Smart Compaing, Fondo Multilateral de Inversiones Miembro del grupo BID.

Kliksberg, B. "Repensando el Estado para el desarrollo social; más allá de convencionalismos y dogmas" en Reforma y Democracia, Revista del CLAD 8, 1997. https://doi.org/10.22201/fcpys.2448492xe.1998.173.491 63
León et al, (2012) “La innovación social en el contexto de la responsabilidad social empresarial”. Forum Empresarial, vol. 17, núm I San Juan (Puerto Rico): Centro de investigaciones Comerciales en Iniciativas Académicas. Pp 3 I-63. https://doi.org/10.33801/fe.v17i1.3745

ONU (Organización de las Naciones Unidas) (2000a) “Objetivos de desarrollo del milenio", Nueva York, Estado Unidos de América.

Schvarstein, L. (2003) "La Inteligencia social en las organizaciones" Desarrollando las competencias necesarias para el ejercicio efectivo de la responsabilidad social, Editorial Paidós, Buenos Aires, Argentina.

STPF (Social Performance Task Force) (2012) [página web]. Consultado: 18 de septiembre de 2019. Disponible en https:// sptf.info/universal-standards-for-spm/universalstandards

STPF (Social Performance Task Force) (2019) [página web]. Consultado: 14 de septiembre de 2019. Disponible en https://sptf.info/universal-standards-for-spm/start-here

Wardle, L. (20/4) “Guía para la implementación de los estándares universales para la gestión del desempeño social" social performance task forcé.

\section{Sobre autor}

\section{Marvin Amilcar Aldana Bolaños}

Es Contador Público y Auditor egresado de la Universidad Mariano Gálvez de Guatemala, 12 años de experiencia en auditoría interna; Maestría en Gerencia Administrativa en Recursos Humanos (Summa Cum Laude), por la Universidad de San Carlos de Guatemala, conferencista invitado para la disertación del tema gestión del talento humano hacia una nueva perspectiva para las competencias a estudiantes de ingeniería en sistemas de la Universidad Mariano Gálvez sede Jutiapa. 
Copyright (c) 2020 Marvin Amilcar Aldana Bolaños

\section{(a) (1)}

Este texto está protegido por una licencia Creative Commons 4.0.

Usted es libre para Compartir -copiar y redistribuir el material en cualquier medio o formato - y Adaptar eldocumento - remezclar, transformar y crear a partir del material- para cualquier propósito, incluso comercialmente, siempre que cumpla la condición de:

Atribución: Usted debe reconocer el crédito de una obra de manera adecuada, proporcionar un enlace a la licencia,e indicar si se han realizado cambios. Puede hacerlo en cualquier forma razonable, pero no de forma tal que sugieraque tiene el apoyo del licenciante o lo recibe por el uso que hace.

Resumen de licencia - Texto completo de la licencia 